\title{
Impacto das Ações de contenção à Covid-19 em Bolsas de Valores de Países Desenvolvidos e em Desenvolvimento
}

\author{
Haroldo R. Vieira Jr ${ }^{1}$, Luiz Gomes-Jr ${ }^{1}$ \\ ${ }^{1}$ Universidade Tecnológica Federal do Paraná (UTFPR) - Curitiba - Brazil \\ haroldo@alunos.utfpf.edu.br, lcjunior@utfpr.edu.br
}

\begin{abstract}
This paper assesses how government actions against COVID-19 influenced global markets. 14 countries, developed and emerging, were selected, and the performance of each country's indices and government measures were compared. The analyzed actions were the ones that promoted social distancing, closing international borders and emergency assistance. Linear regression was used to assess whether there was a significant impact up to five days after the release or execution of the measures. In most cases, there was no significant relationship between exchanges and government actions, but most days where there was significance, the impact was positive.
\end{abstract}

Resumo. Para analisar como essas ações governamentais de combate ao COVID-19 afetaram os mercados financeiros mundiais, foram selecionados 14 países, desenvolvidos e emergentes, e se fez a associação entre o desempenho dos respectivos índices de bolsa de cada país com as medidas governamentais. Foram analisadas as ações referentes a distanciamento social, fechamento de fronteiras internacionais e auxílio emergencial. Utilizou-se regressão linear para avaliar se houve impacto significativo até cinco dias após a divulgação ou execução das medidas. Na maioria dos casos, não houve relação significante entre as bolsas e as ações governamentais, porém a maioria dos dias em que houve significância, o impacto foi positivo.

\section{Introdução}

Para combater a transmissão do novo coronavírus, várias medidas não farmacológicas foram impostas pelos governos de vários países, entre elas: proibir aglomerações de pessoas, proibir o trânsito de pessoas entre países (fechamento de fronteiras), fechamento de serviços não essenciais e, em última medida, lockdown total. [Flaxman et al. 2020] demonstraram que o lockdown bem feito conseguia reduzir significativamente a taxa de contágio. [Brauner et al. 2021] estudaram como cada medida de distanciamento foi capaz de reduzir a taxa de contágio, mostrando que as ações eram necessárias para frear a disseminação do vírus e para instauração de períodos de estabilidade.

Entretanto, conforme concluem [Baker et al. 2020], essas medidas fizeram com que vários setores econômicos perdessem parcialmente (ou temporariamente) a capacidade de funcionar e, consequentemente, gerar renda. [Liu et al. 2020] concluem que notícias relacionadas ao alastramento da pandemia pelo mundo foram significativas para gerar choques em vários mercados de bolsa no mundo devido à incerteza quanto ao impacto da doença nos mercados, sendo um dos medos a paralização que ocorreu na China. 
Portanto, é necessário compreender como as medidas de combate ao Sars-Cov-2 foram entendidas pelos mercados mundiais sabendo-se que elas seriam prejudiciais para a economia como um todo no curto prazo, porém ajudariam a controlar a crise, evitando colapso do sistema de saúde e salvando vidas.

Este trabalho analisa como o mercado reage aos choques provocados por ações governamentais não farmacológicas de combate ao coronavírus. Para verificar como isso pode ocorrer, é necessário relacionar as variações do mercado aos anúncios e ações efetivas dos governos dos países estudados. Esta relação é feita verificando se a existência e intensidade do impacto é estatisticamente relevante nos retornos das bolsas de valores imediatamente após os anúncios governamentais.

São usados os principais índices de bolsa de valores de cada região, sendo retratados como séries temporais. Para verificar a relação estatística entre estas séries temporais é utilizado o método de regressão linear. São adicionadas outras variáveis como as cotações do petróleo WTI, volatilidade e os dias da semana para controle dos efeitos que podem ser causados por estas variáveis nos valores dos retornos. O impacto a ser analisado é de como as medidas afetaram as bolsas durante cinco dias úteis seguidos, de acordo com calendário financeiro de cada país.

O código foi redigido em linguagem python e a regressão linear foi feita utilizando-se a biblioteca statsmodels, especificamente a função OLS (Ordinary Least Squares) ${ }^{1}$.

O restante deste artigo está organizado da seguinte forma: a seção 2 apresenta a revisão da literatura, mostrando trabalhos relacionados ao artigo atual; a seção 3 introduz a metodologia, sendo informando quais os dados e tratamentos usados, modelo matemático e método de análise; a seção 4 é apresenta os resultados; a seção 5 conclui o estudo.

\section{Revisão da Literatura}

Com o objetivo de avaliar os impactos de medidas governamentais, modelos de regressão linear são usados em [Mohler et al. 2020] para verificar qual a relação entre a variação da criminalidade nas cidades de Los Angeles e Indianápolis e os períodos de quarentena impostos em cada uma das cidades. $\mathrm{O}$ estudo faz isso com duas formas, sendo a primeira usando como variável dependente a quantidade de denúncias por telefone em função de três variáveis indicando se o período é durante a quarentena, os dias da semana e o mês. A segunda utiliza a mesma variável dependente, porém substitui-se a variável indicativa de quarentena pelos dados de mobilidade do Google. O estudo mostra que houve impacto relevante estatisticamente em alguns tipos de crime, como o número de roubos em sinais de trânsito diminuindo e a violência doméstica e roubos em geral aumentando, principalmente em Los Angeles.

A análise da variação do mercado de capitais em função do número de casos e mortes em um país foi abordada por [Ashraf 2020b] . Neste estudo a regressão é feita separadamente para morte e para casos, sendo que em cada cálculo são usadas variáveis representativas de cada país e variáveis para controlar possíveis características a nível local e global, respectivamente. O estudo concluiu que até a data de 17 de abril de 2020

\footnotetext{
${ }^{1}$ Disponível pelo link: https://www.statsmodels.org
} 
havia relação, na maioria dos países, entre a queda das bolsas e o aumento do número de casos, porém essa relação não existia para o número de mortes.

Em [Ashraf 2020a] e em [Narayan et al. 2021], a regressão linear é usada para verificar o impacto das intervenções governamentais nos mercados financeiros usando-se a variação dos retornos dos índices de bolsa. Neste são usadas variáveis indicando as datas em que foram realizadas as ações governamentais dos países que pertencem ao G7 e, naquele, são usados os dados de 77 países em função, principalmente, dos casos confirmados de COVID-19 e dos índices de respostas governamentais do website OxCGRT 2. [Ashraf 2020a] conclui que os impactos de divulgações de medidas de distanciamento social podem ser tanto positivas (de forma indireta ao diminuir os casos em um prazo maior) quanto negativas (de forma direta). Além disso, mostra que, em geral, programas de conscientização sobre o COVID-19 e pacotes de estimulo possuem impacto positivo. [Narayan et al. 2021] mostram que o agregado de ações referentes a estabelecimento de lockdown, criação de um pacote de estímulos e proibição de voos internacionais, tiveram um impacto positivo nos mercados na recuperação econômica. Entretanto, o estudo também mostra impactos positivos e negativos a depender do país para as medidas de lockdown.

As metodologias de estudo de eventos são comumente usadas para avaliar o impacto de alguma informação nova no mercado [Bodie et al. 2015], sendo que as metodologias de média dos retornos anormais (AAR) e média dos retornos anormais cumulativos (CAAR) são usadas em [Liu et al. 2020] para avaliar se as notícias sobre o alastramento das infecções pelo novo coronavírus geravam algum impacto nas maiores bolsas de valores do mundo. $\mathrm{O}$ estudo demonstrou que o surto de coronavírus teve um forte impacto negativo nas áreas afetadas, sendo que duas quedas significativas são observadas com o AAR e o CAAR 1 e 24 dias após a informação sobre a transmissibilidade entre humanos, sendo o primeiro impacto na região asiática e o segundo fora da região asiática (surto da Itália amplamente divulgado).

A recuperação dos mercados de bolsas de valores após pandemias foi analisada em [David et al. 2021] usando o modelo de série temporal Vector Error Corretion (VEC). O estudo verificou que os choques de pandemias nos mercados tiveram grande impacto negativo no surto inicial, porém, para a pandemia de COVID-19, a duração da volatilidade alta e manutenção do impacto negativo foi mais duradoura que nas outras pandemias analisadas (EBOLA, MERS e SARS). Este estudo também constatou a péssima recuperação do índice Ibovespa durante o período analisado, sugerindo maior risco atrelado a países com maior fragilidade econômica e social.

O presente artigo se baseia na metodologia aplicada no trabalho de [Narayan et al. 2021], porém difere deste por analisar adicionalmente os países emergentes, utilizar os índices de respostas governamentais do website OxCGRT para definir as datas de estabelecimento das ações de combate ao COVID-19.

\footnotetext{
${ }^{2}$ Disponível pelo link: https://github.com/OxCGRT/covid-policy-tracke
} 


\section{Metodologia}

\subsection{Descrição e tratamento dos dados}

Para medir o impacto das medidas governamentais no mercado financeiro de cada país, foram utilizadas as cotações de fechamento do principal índice de cada país analisado: África do sul (FTSE/JSE Top 40), Alemanha (DAX), Brasil (Ibovespa), Canada (S\&P/TSX), Estados Unidos (S\&P 500), França (CAC 40), Índia (BSE Sensex), Indonésia (IDX Composite), Itália (FTSE MIB), Japão (NIKKEI 225), México (S\&P/BMV IPC), Reino Unido (FTSE 100), Rússia (MOEX), Turquia (BIST 100). Foram incluídas também as cotações de preço do petróleo futuro WTI entre os períodos de 01/07/2019 e 01/05/2020. As cotações foram obtidas no site investing.com.

As datas das medidas governamentais foram estimadas de acordo com os dados disponibilizados pela Universidade de Oxford pelo seu programa "COVID-19 Government Response Tracker"3. Especificamente, foram utilizados os índices "C6 Stay at home requirements", "C8 International travel controls" e "E1 Income support". Tais índices foram escolhidos como forma de aproximar as datas das medidas governamentais referentes à imposição de medidas restritivas de movimentação local, restrições de viagens internacionais e auxílio financeiro emergencial, respectivamente. Estas medidas foram escolhidas por se entender que são as mais impactantes para a variação dos índices financeiros.

Os dados de cotação de índices de bolsas e do preço do petróleo futuro foram utilizados para calcular o retorno diário em percentual. As cotações de índice de cada país obedecem a um calendário próprio, sendo que alguns podem conter mais dias úteis que outros. Foram excluídos os dias não úteis de cada uma das séries temporais de retornos dos índices de cada país, sendo que um dia útil em um país não impactaria em outro país. Dessa forma, cada país teve uma série temporal em função de suas datas, havendo até mesmo tamanhos diferentes de séries temporais. Utilizando-se das séries temporais de retornos, foi criado para cada índice uma série temporal de volatilidade a partir do modelo Garch(1,1) [Neves and Rossi 2014].

Para definir quais datas seriam utilizadas para cada medida governamental, foram utilizadas as datas em que os indicadores observados atingiram o seu valor máximo dentro do período observado. Caso a data observada não esteja entre as datas da série temporal dos retornos, a data seria transferida para a data útil imediatamente anterior, dessa forma facilitando gerar as variáveis dummy para a regressão a ser usada no método.

A Tabela 1 mostra que cada país teve um nível de resposta diferente para indicador de medida governamental medido por Oxford, sendo que alguns países não realizaram nenhuma ação governamental em alguns dos índices de resposta.

\subsection{Modelo Estatístico}

A abordagem utilizada neste trabalho é o uso de regressões lineares múltiplas usando os retornos observados do ativo financeiro a cada fechamento em função de outras variáveis, conforme:

$$
r_{t}=\sum_{i=0}^{n} \beta_{i} \cdot X_{i, t^{\prime}}+\epsilon_{t}
$$

\footnotetext{
${ }^{3}$ Disponível pelo link: https://github.com/OxCGRT/covid-policy-tracke
} 
Tabela 1. Grau dos índices C6, C8 e E1 por país.

\begin{tabular}{|l|l|l|l|l|l|l|}
\hline \multirow{2}{*}{ País } & C6 & \multicolumn{2}{l}{ C8 } & \multicolumn{2}{l|}{ E1 } \\
\cline { 2 - 7 } & Data & Índice & Data & Índice & Data & Índice \\
\hline Brasil & $13 / 3$ & 1.0 & $27 / 3$ & 4.0 & $2 / 4$ & 1.0 \\
\hline Canadá & $14 / 3$ & 1.0 & $18 / 3$ & 4.0 & $15 / 3$ & 2.0 \\
\hline França & $17 / 3$ & 2.0 & $17 / 3$ & 3.0 & $16 / 3$ & 2.0 \\
\hline Alemanha & $21 / 3$ & 2.0 & $18 / 3$ & 4.0 & $16 / 3$ & 2.0 \\
\hline Índia & $22 / 3$ & 3.0 & $22 / 3$ & 4.0 & $20 / 3$ & 1.0 \\
\hline Indonesia & $10 / 4$ & 2.0 & $19 / 3$ & 4.0 & $1 / 1$ & 0.0 \\
\hline Itália & $20 / 3$ & 3.0 & $30 / 1$ & 3.0 & $17 / 3$ & 1.0 \\
\hline Japão & $7 / 4$ & 1.0 & $1 / 2$ & 3.0 & $16 / 4$ & 1.0 \\
\hline México & $30 / 3$ & 2.0 & $21 / 3$ & 3.0 & $1 / 1$ & 0.0 \\
\hline Rússia & $30 / 3$ & 3.0 & $30 / 3$ & 4.0 & $1 / 1$ & 0.0 \\
\hline África do Sul & $26 / 3$ & 2.0 & $26 / 3$ & 4.0 & $21 / 4$ & 1.0 \\
\hline Turquia & $11 / 4$ & 3.0 & $27 / 3$ & 4.0 & $7 / 4$ & 2.0 \\
\hline Reino Unido & $22 / 3$ & 2.0 & $1 / 1$ & 0.0 & $20 / 3$ & 2.0 \\
\hline Estados Unidos & $15 / 3$ & 2.0 & $2 / 3$ & 3.0 & $27 / 3$ & 2.0 \\
\hline
\end{tabular}

Em que $r_{t}$ é o retorno no tempo $t, \beta_{i}$ é o coeficiente de regressão linear referente a uma variável exógena $i$ (incluso o intercepto), $X_{i, t^{\prime}}$ é a variável exógena $i$ no tempo $t^{\prime}$, e $\epsilon_{t}$ é o resíduo da regressão no tempo $t$.

Para gerar os valores de beta e estimar o impacto de cada variável exógena nos retornos, o método dos mínimos quadrados ordinários é usado neste trabalho [Neves and Rossi 2014]. Ao se gerar os coeficientes, também são gerados os valores das variâncias destes coeficientes e feito o teste estatístico de $t$-student, sendo gerados os $p$-values para verificar se os valores são estatisticamente significantes.

\subsection{Método de análise}

Para avaliar o impacto das medidas governamentais em cada índice, baseou-se no método apresentado em [Narayan et al. 2021]. Foi avaliado como a nova notícia foi percebida pelos investidores em um prazo de 5 dias, levando em conta as variações dos retornos, volatilidade, preço do petróleo e dia da semana. O modelo de regressão é como disposto em:

$$
\begin{aligned}
\text { Return }_{t}= & \alpha+\sum_{i=1}^{5} \beta_{i} \text { OOVT }_{t-i}+\beta_{6} O I L_{t-1}+\beta_{7} \text { Return }_{t-1}+ \\
& \beta_{8} \text { VOL }_{t-1}+\beta_{9} M O N_{t-1}+\beta_{10} T U E_{t-1}+\beta_{11} W_{E D_{t-1}+} \\
& \beta_{12} \text { THU }_{t-1}+\beta_{13} \text { FRI }_{t-1}+\epsilon_{t}
\end{aligned}
$$

sendo Return $n_{t}$ o retorno de cada índice, GOVT $T_{t}$ uma variável dummy representando o momento em que determinada medida governamental foi implementada, $O I L_{t}$ o retorno sobre o preço do Petróleo WTI, $V O L_{t}$ a volatilidade dos retornos gerada pelos retornos diários usando-se modelo GARCH(1,1) e, $M O N_{t}, T U E_{t}, W E D_{t}, T H U_{t}$ e $F R I_{t}$ são as variáveis dummy indicando os dias da semana na ordem de segunda à sexta-feira.

Cada regressão é feita para cada índice de bolsa e medida governamental, totalizando 42 regressões ao todo. Como cada regressão possui 13 termos e cada regressão uti- 
Tabela 2. Coeficientes de regressão linear referentes ao índice C6 (medidas de distanciamento social) e, em parênteses, seus respectivos $p$-values.

\begin{tabular}{|c|c|c|c|c|c|}
\hline País & C6 $(t=-1)$ & C6 $(t=-2)$ & C6 $(t=-3)$ & C6 $(t=-4)$ & C6 $(t=-5)$ \\
\hline Brasil & $\begin{array}{c}-13,86185^{*} \\
(0,00004)\end{array}$ & $\begin{array}{c}1,07934 \\
(0,72477)\end{array}$ & $\begin{array}{c}-11,39893^{*} \\
(0,00013)\end{array}$ & $\begin{array}{l}-0,34484 \\
(0,90619)\end{array}$ & $\begin{array}{l}-1,89450 \\
(0,50341)\end{array}$ \\
\hline Rússia & $\begin{array}{c}3,14503 \\
(0,06637)\end{array}$ & $\begin{array}{l}-1,47561 \\
(0,39004)\end{array}$ & $\begin{array}{c}2,98465 \\
(0,07887)\end{array}$ & $\begin{array}{c}1,23028 \\
(0,47231)\end{array}$ & $\begin{array}{c}2,10737 \\
(0,21365)\end{array}$ \\
\hline Índia & $\begin{array}{c}0,94377 \\
(0,68776)\end{array}$ & $\begin{array}{l}8,85352 * \\
(0,00004)\end{array}$ & $\begin{array}{l}7,91888 * \\
(0,00035)\end{array}$ & $\begin{array}{c}1,79447 \\
(0,39562)\end{array}$ & $\begin{array}{l}-3,06140 \\
(0,12970)\end{array}$ \\
\hline África do Sul & $\begin{array}{l}-4,30031 \\
(0,05021)\end{array}$ & $\begin{array}{c}0,97910 \\
(0,63414)\end{array}$ & $\begin{array}{c}1,59152 \\
(0,43097)\end{array}$ & $\begin{array}{l}-1,88497 \\
(0,34861)\end{array}$ & $\begin{array}{c}2,41730 \\
(0,22560)\end{array}$ \\
\hline México & $\begin{array}{c}1,31967 \\
(0,37640)\end{array}$ & $\begin{array}{l}-2,27309 \\
(0,12535)\end{array}$ & $\begin{array}{c}0,42532 \\
(0,77384)\end{array}$ & $\begin{array}{l}-1,37773 \\
(0,34968)\end{array}$ & $\begin{array}{l}4,57589 * \\
(0,00209)\end{array}$ \\
\hline Turquia & $\begin{array}{c}1,78243 \\
(0,28928)\end{array}$ & $\begin{array}{l}-2,61706 \\
(0,12161)\end{array}$ & $\begin{array}{c}0,40737 \\
(0,80955)\end{array}$ & $\begin{array}{c}2,14713 \\
(0,20218)\end{array}$ & $\begin{array}{c}1,34146 \\
(0,42694)\end{array}$ \\
\hline Indonesia & $\begin{array}{c}1,63993 \\
(0,29586)\end{array}$ & $\begin{array}{l}-1,83115 \\
(0,24466)\end{array}$ & $\begin{array}{l}-2,86651 \\
(0,06862)\end{array}$ & $\begin{array}{c}3,90568 \\
(0,01428)\end{array}$ & $\begin{array}{l}-1,09814 \\
(0,48941)\end{array}$ \\
\hline Alemanha & $\begin{array}{c}11,11713 * \\
(0,00000)\end{array}$ & $\begin{array}{c}2,89974 \\
(0,17552)\end{array}$ & $\begin{array}{c}2,35492 \\
(0,21110)\end{array}$ & $\begin{array}{l}-2,99093 \\
(0,10747)\end{array}$ & $\begin{array}{c}2,81235 \\
(0,12659)\end{array}$ \\
\hline França & $\begin{array}{l}-5,85747 * \\
(0,00157)\end{array}$ & $\begin{array}{c}3,95007 \\
(0,03255)\end{array}$ & $\begin{array}{c}4,95685 \\
(0,00712)\end{array}$ & $\begin{array}{l}-2,95489 \\
(0,11215)\end{array}$ & $\begin{array}{l}8,65791 * \\
(0,00000)\end{array}$ \\
\hline Canadá & $\begin{array}{l}-1,30295 \\
(0,57289)\end{array}$ & $\begin{array}{c}-7,52235^{*} \\
(0,00051)\end{array}$ & $\begin{array}{c}1,49664 \\
(0,49764)\end{array}$ & $\begin{array}{l}-2,07053 \\
(0,32996)\end{array}$ & $\begin{array}{l}-6,01590 \\
(0,00433)\end{array}$ \\
\hline Estados Unidos & $\begin{array}{c}0,58653 \\
(0,80850)\end{array}$ & $\begin{array}{l}-3,07179 \\
(0,16484)\end{array}$ & $\begin{array}{l}-1,69611 \\
(0,43837)\end{array}$ & $\begin{array}{l}-4,69492 \\
(0,02716)\end{array}$ & $\begin{array}{l}-4,55517 \\
(0,03284)\end{array}$ \\
\hline Itália & $\begin{array}{c}0,44166 \\
(0,83587)\end{array}$ & $\begin{array}{l}8,92945^{*} \\
(0,00003)\end{array}$ & $\begin{array}{c}4,23612 \\
(0,06714)\end{array}$ & $\begin{array}{c}1,87672 \\
(0,38087)\end{array}$ & $\begin{array}{l}-2,25655 \\
(0,28723)\end{array}$ \\
\hline Japão & $\begin{array}{c}2,00955 \\
(0,22250)\end{array}$ & $\begin{array}{l}-0,22094 \\
(0,89305)\end{array}$ & $\begin{array}{c}0,00000 \\
(0,01314)\end{array}$ & $\begin{array}{l}-2,11644 \\
(0,19523)\end{array}$ & $\begin{array}{c}3,14607 \\
(0,05555)\end{array}$ \\
\hline Reino Unido & $\begin{array}{l}9,08710^{*} \\
(0,00000)\end{array}$ & $\begin{array}{l}6,42836^{*} \\
(0,00083)\end{array}$ & $\begin{array}{c}4,03084 \\
(0,02181)\end{array}$ & $\begin{array}{c}-4,19091 \\
(0,01318)\end{array}$ & $\begin{array}{c}1,73691 \\
(0,29881)\end{array}$ \\
\hline
\end{tabular}

liza amostras distintas, foi feito o ajuste de Bonferroni simples, [Bland and Altman 1995], gerando alpha de valor igual a 0.0038 como limiar de relevância estatística.

\section{Resultados}

As Tabelas 2, 3 e 4 demonstram os coeficientes de regressão para cada índice de ação governamental escolhido e seus respectivos p-values de acordo com a medida de contenção.

Para o índice C6, que indica os esforços do governo para manter a população em distanciamento social, a Tabela 2 mostra que de 70 coeficientes apenas 12 são estatisticamente significantes, sendo que 4 são negativos e os outros 7 são positivos, não havendo diferença entre os países emergentes e desenvolvidos. Dentre os coeficientes estatisticamente relevantes, somente a França teve valores com sinais trocados e o Brasil teve os valores negativos com maior módulo, indicando forte impacto negativo. Ao se comparar o grau do índice e a data escolhida por país separadamente, percebe-se que não há relação clara, porém ao se observar os Brasil-Canada e Alemanha-Reino Unido, em que cada par teve mesmo grau de intensidade do indicador e a data da política pública teve apenas 1 dia de diferença, percebe-se que seus coeficientes relevantes tiveram sinais similares.

Conforme Tabela 3, para o índice C8, que mede as restrições de viagens interna- 
Tabela 3. Coeficientes de regressão linear referentes ao índice C8 (restrições de viagens internacionais) e, em parênteses, seus respectivos $p$-values.

\begin{tabular}{|l|l|l|l|l|l|}
\hline País & $\mathbf{C 8}(\mathbf{t}=\mathbf{- 1})$ & $\mathbf{C 8}(\mathbf{t}=-\mathbf{2})$ & $\mathbf{C 8}(\mathbf{t}=-\mathbf{3})$ & $\mathbf{C 8}(\mathbf{t}=-\mathbf{4})$ & $\mathbf{C 8}(\mathbf{t}=\mathbf{- 5})$ \\
\hline Brasil & 1,23032 & $-2,02912$ & $-3,18897$ & 1,58096 & $-2,46362$ \\
& $(0,66853)$ & $(0,47704)$ & $(0,26341)$ & $(0,57739)$ & $(0,39125)$ \\
\hline Rússia & 3,14503 & $-1,47561$ & 2,98465 & 1,23028 & 2,10737 \\
& $(0,06637)$ & $(0,39004)$ & $(0,07887)$ & $(0,47231)$ & $(0,21365)$ \\
\hline Índia & 0,94377 & $8,85352^{*}$ & $7,91888^{*}$ & 1,79447 & $-3,06140$ \\
& $(0,68776)$ & $(0,00004)$ & $(0,00035)$ & $(0,39562)$ & $(0,12970)$ \\
\hline África do Sul & $-4,30031$ & 0,97910 & 1,59152 & $-1,88497$ & 2,41730 \\
& $(0,05021)$ & $(0,63414)$ & $(0,43097)$ & $(0,34861)$ & $(0,22560)$ \\
\hline México & $5,72767^{*}$ & 3,52798 & 1,51496 & $-4,76154^{*}$ & 2,69182 \\
& $(0,00008)$ & $(0,01775)$ & $(0,29295)$ & $(0,00083)$ & $(0,06666)$ \\
\hline Turquia & 0,70309 & 1,77889 & $-0,23305$ & 0,71931 & 0,11266 \\
& $(0,68643)$ & $(0,30115)$ & $(0,89213)$ & $(0,67354)$ & $(0,94745)$ \\
\hline Indonesia & 3,34500 & $-3,92519$ & $-0,37386$ & $11,15456^{*}$ & 4,56402 \\
& $(0,01832)$ & $(0,00433)$ & $(0,78755)$ & $(0,00000)$ & $(0,00576)$ \\
\hline Alemanha & 3,38511 & 4,39611 & $-0,97903$ & $11,24031^{*}$ & 3,28628 \\
& $(0,06943)$ & $(0,01804)$ & $(0,59759)$ & $(0,00000)$ & $(0,12334)$ \\
\hline França & $-5,85747$ & 3,95007 & 4,95685 & $-2,95489$ & $8,65791^{*}$ \\
& $(0,00157)$ & $(0,03255)$ & $(0,00712)$ & $(0,11215)$ & $(0,00000)$ \\
\hline Canadá & 2,64839 & 0,12488 & $-5,09858$ & $10,63675^{*}$ & $11,41452^{*}$ \\
& $(0,17656)$ & $(0,94816)$ & $(0,00683)$ & $(0,00000)$ & $(0,00000)$ \\
\hline Estados Unidos & $-1,11333$ & 3,02658 & $-1,60025$ & $-3,08908$ & $-8,03542^{*}$ \\
& $(0,58374)$ & $(0,13376)$ & $(0,43000)$ & $(0,12673)$ & $(0,00009)$ \\
\hline Itália & $-2,33285$ & 1,19883 & 1,45860 & 1,81138 & 1,42706 \\
& $(0,28988)$ & $(0,58660)$ & $(0,50792)$ & $(0,41099)$ & $(0,51710)$ \\
\hline Japão & 0,22926 & 1,02667 & 2,46329 & $-0,60342$ & $-0,25021$ \\
& $(0,88861)$ & $(0,53023)$ & $(0,13328)$ & $(0,71407)$ & $(0,87863)$ \\
\hline Reino Unido & 0,38686 & $-0,38254$ & $-0,40350$ & 0,03452 & 0,70253 \\
& $(0,83208)$ & $(0,83386)$ & $(0,82492)$ & $(0,98490)$ & $(0,70010)$ \\
\hline
\end{tabular}

cionais, apenas 10 coeficientes são estatisticamente relevantes, sendo que 8 são positivos, não havendo diferença entre os países desenvolvidos e emergentes quanto ao sinal dos coeficientes. Para este indicador, somente o México teve coeficientes relevantes com o sinal trocado. Percebe-se que o Reino Unido não tomou nenhuma atitude em relação a fechamento das fronteiras durante o período estudado, ficando evidente o motivo dos $p$ values para este país e índice ficarem tão grandes quando comparado com outros países. Da mesma forma que ocorreu para o índice $\mathrm{C} 8$, percebe-se que os graus e as datas não tiveram forte influência sobre os coeficientes de forma separada, porém ao se observar os países Alemanha, Canada e Indonesia, percebe-se que os coeficientes estatisticamente relevantes possuem o mesmo sinal e intensidade similar, além de serem em dias próximos. Ao se analisar os países Turquia, África do Sul e Brasil, que possuem características similares na Tabela 1, não se identifica coeficientes significantes.

Para o índice E1, que mede o grau do auxílio financeiro, somente 8 coeficientes foram considerados significativos (5 positivos e 3 negativos), sendo que a Índia tive 3 (2 positivos e um negativo). A data do início do auxílio emergencial do Reino Unido é igual a da Índia, porém possui maior grau. Para o Reino Unido, aparentemente, o auxílio trouxe retornos positivos para a bolsa de valores. Rússia, México e Indonésia não lançaram nenhum programa de auxílio econômico e, consequentemente, não tiveram nenhum co- 
Tabela 4. Coeficientes de regressão linear referentes ao índice E1 (auxílio financeiro) e, em parênteses, seus respectivos p-values.

\begin{tabular}{|l|l|l|l|l|l|}
\hline Pais & $\mathbf{E} 1(\mathbf{t}=\mathbf{- 1})$ & $\mathbf{E 1}(\mathbf{t}=-2)$ & $\mathbf{E} \mathbf{( t}=-\mathbf{3})$ & $\mathbf{E}(\mathbf{t}=-\mathbf{4})$ & $\mathbf{E 1}(\mathbf{t}=\mathbf{- 5})$ \\
\hline Brasil & $-2,31468$ & 6,62349 & 5,07868 & 4,28287 & 0,50161 \\
& $(0,40972)$ & $(0,01852)$ & $(0,07508)$ & $(0,12503)$ & $(0,85678)$ \\
\hline Rússia & 0,21648 & 1,01236 & 0,21795 & 0,43645 & 1,05979 \\
& $(0,89937)$ & $(0,55366)$ & $(0,89854)$ & $(0,79833)$ & $(0,53565)$ \\
\hline Índia & $-11,39941^{*}$ & 1,59569 & $7,87631^{*}$ & $6,40626^{*}$ & 0,56395 \\
& $(0,00000)$ & $(0,45887)$ & $(0,00007)$ & $(0,00173)$ & $(0,77276)$ \\
\hline África do Sul & 2,79236 & 2,76465 & 0,03871 & 0,77852 & 1,99411 \\
& $(0,18940)$ & $(0,16393)$ & $(0,98442)$ & $(0,69210)$ & $(0,31066)$ \\
\hline México & 0,39523 & $-0,08110$ & $-1,01662$ & 0,62226 & 0,47484 \\
& $(0,79371)$ & $(0,95698)$ & $(0,49882)$ & $(0,67928)$ & $(0,75213)$ \\
\hline Turquia & 1,11852 & 2,89018 & 0,00000 & 0,43374 & 1,78661 \\
& $(0,50616)$ & $(0,08701)$ & $(0,15956)$ & $(0,79653)$ & $(0,28853)$ \\
\hline Indonesia & 0,49703 & $-0,42781$ & 0,22751 & $-0,75315$ & 1,00003 \\
& $(0,75746)$ & $(0,79033)$ & $(0,88763)$ & $(0,63997)$ & $(0,53432)$ \\
\hline Alemanha & 2,34115 & $-5,55981$ & 3,04432 & 3,53928 & $-1,81892$ \\
& $(0,24547)$ & $(0,00529)$ & $(0,12979)$ & $(0,07384)$ & $(0,35763)$ \\
\hline França & 3,27494 & $-5,95681^{*}$ & 3,80959 & 4,84287 & $-3,01382$ \\
& $(0,09339)$ & $(0,00222)$ & $(0,05030)$ & $(0,01229)$ & $(0,12320)$ \\
\hline Canadá & $-1,30295$ & $-7,52235^{*}$ & 1,49664 & $-2,07053$ & $-6,01590$ \\
& $(0,57289)$ & $(0,00051)$ & $(0,49764)$ & $(0,32996)$ & $(0,00433)$ \\
\hline Estados Unidos & 2,64924 & $-0,16922$ & $-5,23333$ & 0,60528 & $-0,70899$ \\
& $(0,20773)$ & $(0,93561)$ & $(0,01240)$ & $(0,77259)$ & $(0,73351)$ \\
\hline Itália & 0,16366 & 3,48235 & 2,83637 & 0,38717 & $8,95812^{*}$ \\
& $(0,94111)$ & $(0,10830)$ & $(0,19112)$ & $(0,85623)$ & $(0,00003)$ \\
\hline Japão & 3,35066 & $-1,05783$ & 9,31644 & 4,37074 & 0,39598 \\
& $(0,04049)$ & $(0,52258)$ & $(0,18745)$ & $(0,17923)$ & $(0,82992)$ \\
\hline Reino Unido & $-3,14155$ & $8,98932^{*}$ & $6,17624^{*}$ & 3,80414 & $-4,37418^{*}$ \\
& $(0,05177)$ & $(0,00000)$ & $(0,00124)$ & $(0,02942)$ & $(0,00929)$ \\
\hline
\end{tabular}

eficiente estatisticamente significante e os seus respectivos $p$-values foram elevados em comparação aos outros que tiveram algum grau.

\section{Conclusão}

O presente trabalho foca em avaliar se as medidas governamentais não farmacêuticas de combate ao novo coronavírus produziram efeitos positivos nos mercados financeiros desenvolvidos e em desenvolvimento até final de abril de 2020. Ao serem analisados os impactos nos retornos dos principais índices até 5 dias após as ações governamentais, a maioria dos retornos não sofreu impacto significativo por causa das ações governamentais, porém, dentre os impactos significativos, a maioria foi positiva, sendo 8 em 12 para as ações de distanciamento social, 8 de 10 para fechamento de fronteiras e 5 de 8 para auxílios financeiros, o que indica que as medidas, quando significativas ao mercado, são avaliadas de forma positiva.

Quanto a diferenciar os impactos entre países desenvolvidos e em desenvolvimento, não foi observado nenhum resultado que permitisse inferir alguma diferença, exceto de que a maioria dos coeficientes significativos é de países desenvolvidos $(56,67 \%)$, especialmente ao se analisar o último indicador de auxílio financeiro emergencial $(62,5 \%)$. Este último resultado ocorre, provavelmente, porque três dos países em desen- 
volvimento escolhidos não haviam anunciado oficialmente nenhuma política de auxílio financeiro durante o período estudado.

Entretanto, é necessário estudo mais aprofundado sobre estes impactos, pois alguns fatores podem atrapalhar o desempenho como a correlação intrínseca entre os retornos das bolsas de valores utilizadas e o impacto do grau de cada ação governamental estudada.

Para trabalhos futuros, espera-se fazer uma análise mais rigorosa sobre as covariâncias dos índices ao longo do tempo, empregando-se técnicas mais sofisticadas de estudo de evento e também analisando o impacto agregado dos 5 dias de retorno após as medidas governamentais com técnicas de teste de hipótese como o teste de Wald, verossimilhança ou score test.

\section{Referências}

Ashraf, B. N. (2020a). Economic impact of government interventions during the covid-19 pandemic: International evidence from financial markets. Journal of Behavioral and Experimental Finance, 27:100371.

Ashraf, B. N. (2020b). Stock markets' reaction to covid-19: Cases or fatalities? Research in International Business and Finance, 54:101249.

Baker, S. R., Bloom, N., Davis, S. J., Kost, K., Sammon, M., and Viratyosin, T. (2020). The Unprecedented Stock Market Reaction to COVID-19. The Review of Asset Pricing Studies, 10(4):742-758.

Bland, J. M. and Altman, D. G. (1995). Multiple significance tests: the bonferroni method. BMJ, 310(6973):170.

Bodie, Z., Kane, A., and Marcus, A. (2015). Investimentos. Grupo A.

Brauner, J. M., Mindermann, S., Sharma, M., Johnston, D., Salvatier, J., Gavenčiak, T., Stephenson, A. B., Leech, G., Altman, G., Mikulik, V., Norman, A. J., Monrad, J. T., Besiroglu, T., Ge, H., Hartwick, M. A., Teh, Y. W., Chindelevitch, L., Gal, Y., and Kulveit, J. (2021). Inferring the effectiveness of government interventions against covid19. Science, 371(6531).

David, S., Inácio Jr., C., and Tenreiro Machado, J. A. (2021). The recovery of global stock markets indices after impacts due to pandemics. Research in International Business and Finance, 55:101335.

Flaxman, S., Mishra, S., Gandy, A., and et al. (2020). Estimating the effects of nonpharmaceutical interventions on covid-19 in europe.

Liu, H., Manzoor, A., Wang, C., Zhang, L., and Manzoor, Z. (2020). The covid-19 outbreak and affected countries stock markets response. International Journal of Environmental Research and Public Health, 17(8).

Mohler, G., Bertozzi, A. L., Carter, J., Short, M. B., Sledge, D., Tita, G. E., Uchida, C. D., and Brantingham, P. J. (2020). Impact of social distancing during covid-19 pandemic on crime in los angeles and indianapolis. Journal of Criminal Justice, 68:101692.

Narayan, P. K., Phan, D. H. B., and Liu, G. (2021). Covid-19 lockdowns, stimulus packages, travel bans, and stock returns. Finance Research Letters, 38:101732. 
Neves, C. and Rossi, J. (2014). Econometria e Séries Temporais com Aplicações à Dados da Economia Brasileira. Grupo GEN. 